\title{
Two-Parameter Nyquist Pulses with Better Performance
}

\author{
Stylianos D. Assimonis, Michail Matthaiou, Student Member, IEEE, \\ and George K. Karagiannidis, Senior Member, IEEE
}

\begin{abstract}
We present three novel Nyquist (intersymbol interference free) pulses that outperform two sophisticated pulses reported in literature by Bealieu et al. and Assalini and Tonello. The pulses are based on the concept of inner and outer functions, which was recently explored by the authors. Apart from requiring only two design parameters, the proposed pulses offer an enhanced error performance for various values of roll-off factor and timing jitter along with a smaller maximum distortion.
\end{abstract}

Index Terms-Nyquist pulses, intersymbol interference (ISI), matched filters, pulse shaping methods, timing jitter.

\section{ISI-Free Pulses with Two Design Parameters}

\section{A. Motivation and related works}

$\mathbf{T}$ HE rapid growth of digital communications over the last decades imposes better bandwidth reuse and higher error-free data rates. The most popular Nyquist pulse for distortionless transmission without the presence of intersymbol interference (ISI), is the raised-cosine (RC) pulse which is basically a low-pass filter with odd symmetry around a cutoff frequency and has a cosine shaped roll-off portion [1]. Moreover, the so-called Beaulieu or better than raised cosine (BTRC) pulse [2] outperforms the RC pulse in terms of larger eye opening and smaller error rate. Please note that the Beaulieu pulse remains the best pulse known with an explicit time-domain formula. Other superior pulses, that do not have an explicit time-domain expression, have also been devised [3]; in particular, Assalini and Tonello [4] proposed recently two modified Nyquist pulses, namely the flippedhyperbolic secant (fsech) and the flipped-inverse hyperbolic secant (farcsech). The frequency response of the latter is given as

$$
\begin{aligned}
& S_{1}(f)= \\
& \left\{\begin{array}{lr}
1, & |f| \leq B(1-\alpha) \\
1-\frac{1}{2 \alpha B \gamma_{1}} \operatorname{arcsech}\left(\frac{1}{2 \alpha B}(B(1+\alpha)-|f|)\right), \\
\frac{1}{2 \alpha B \gamma_{1}} \operatorname{arcsech}\left(\frac{1}{2 \alpha B}(|f|-B(1-\alpha))\right), \\
0, & B<|f| \leq B(1+\alpha) \\
& B(1+\alpha)<|f|
\end{array}\right.
\end{aligned}
$$

Manuscript received July 17, 2008. The associate editor coordinating the review of this letter and approving it for publication was R. Blum.

S. D. Assimonis and G. K. Karagiannidis are with the Department of Electrical and Computer Engineering, Aristotle University of Thessaloniki, 54 124, Thessaloniki, Greece (e-mail: \{asimonis, geokarag\}@auth.gr).

M. Matthaiou was with The University of Edinburgh, U.K. He is now with the Institute for Circuit Theory \& Signal Processing, Technische Universität München, Arcistr. 21, 80333, Munich, Germany (e-mail: matthaiou@nws.ei.tum.de).

Digital Object Identifier 10.1109/LCOMM.2008.081117 where $\alpha$ denotes the roll-off factor $(0 \leq \alpha \leq 1)$, determining the bandwidth occupied by the pulse and the timedomain tail suppression, $B$ is the Nyquist frequency while $\gamma_{1}=\ln (\sqrt{3}+2) /(\alpha B)$. We note that farcsech is systematically superior than the Beaulieu pulse (smaller SER and maximum distortion in conjunction with an enhanced eye opening). Due to space limitations, in the following comparative study it will be our reference pulse since, to the best of our knowledge, is the best pulse documented in the corresponding literature with only two design parameters (degrees of freedom).

\section{B. Novel improved Nyquist pulses}

In the present contribution, we propose three novel Nyquist pulses which yield an improved performance compared to farcsech and have been constructed upon the notions of inner and outer functions, originally proposed in [5] and recently extended by the authors in [6]. More specifically, the first pulse is the so-called inverse-cosine inverse-hyperbolic sine $(\operatorname{acos}[\operatorname{asinh}])$ whose frequency response reads

$$
\begin{aligned}
& S_{2}(f)= \\
& \left\{\begin{array}{lr}
1, & |f| \leq B(1-\alpha) \\
1-\frac{1}{2 \gamma_{2}} \operatorname{acos}\left(\operatorname{asinh}\left(\frac{\gamma_{0}}{2 \alpha B}(-|f|+B(1+\alpha))\right)\right), \\
\frac{1}{2 \gamma_{2}} \operatorname{acos}\left(\operatorname{asinh}\left(\frac{\gamma_{0}}{2 \alpha B}(+|f|-B(1-\alpha))\right)\right), \\
0, & B<|f| \leq B(1+\alpha) \\
0(1+\alpha)<|f|
\end{array}\right.
\end{aligned}
$$

where $\gamma_{0}, \gamma_{2}$ are constants, defined as

$$
\gamma_{0}=\sinh (1) \text { and } \gamma_{2}=\operatorname{acos}\left(\operatorname{asinh}\left(\frac{\sinh (1)}{2}\right)\right) .
$$

The second pulse, which we will refer to hereafter as inverse-cosine inverse-tangent (acos[atan]) is defined in a similar manner according to

$$
\begin{aligned}
& S_{3}(f)= \\
& \left\{\begin{array}{lr}
1, & |f| \leq B(1-\alpha) \\
1-\frac{1}{2 \gamma_{3}} \operatorname{acos}\left(\operatorname{atan}\left(\frac{\gamma_{0}^{\prime}}{2 \alpha B}(-|f|+B(1+\alpha))\right)\right. & B(1-\alpha)<|f| \leq B \\
\frac{1}{2 \gamma_{3}} \operatorname{acos}\left(\operatorname{atan}\left(\frac{\gamma_{0}^{\prime}}{2 \alpha B}(+|f|-B(1-\alpha))\right)\right), \\
0, & B<|f| \leq B(1+\alpha) \\
B(1+\alpha)<|f|
\end{array}\right.
\end{aligned}
$$




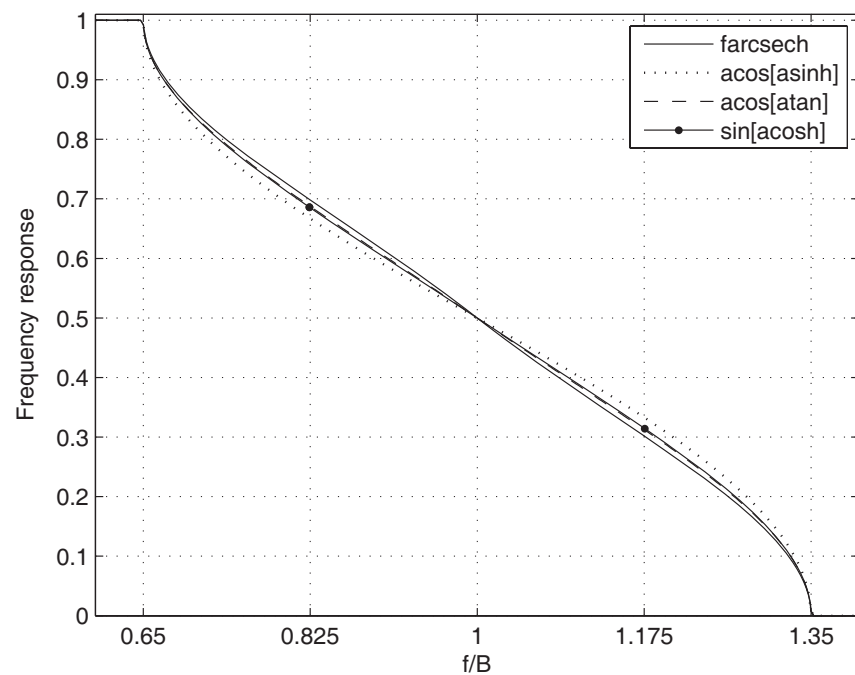

Fig. 1. Frequency responses with roll-off factor $\alpha=0.35$.

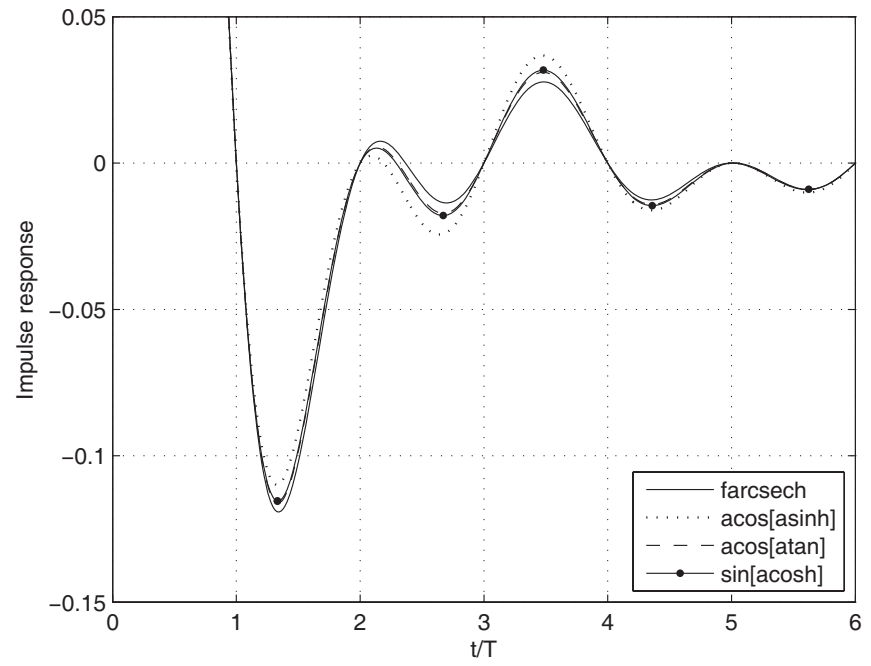

Fig. 2. Impulse responses with roll-off factor $\alpha=0.35$.

where $\gamma_{0}^{\prime}, \gamma_{3}$ are constants, defined as

$$
\gamma_{0}^{\prime}=\tan (1) \text { and } \gamma_{3}=\operatorname{acos}\left(\operatorname{atan}\left(\frac{\tan (1)}{2}\right)\right) .
$$

The third proposed pulse is referred to as sine inversehyperbolic cosine $(\sin [\operatorname{acosh}])$, with a frequency response as $S_{4}(f)=$

$$
\left\{\begin{array}{lr}
1, & |f| \leq B(1-\alpha) \\
1-\frac{1}{2 \gamma_{4}} \sin \left(\operatorname{acosh}\left(\frac{1}{2 \alpha B}(-|f|+B(1+\alpha))\right)\right), \\
\frac{1}{2 \gamma_{4}} \sin \left(\operatorname{acosh}\left(\frac{1}{2 \alpha B}(+|f|-B(1-\alpha))\right)\right), \\
0, & B<|f| \leq B(1+\alpha) \\
& B(1+\alpha)<|f|
\end{array}\right.
$$

where $\gamma_{4}$ is a constant, given by

$$
\gamma_{4}=\sin \left(\operatorname{acosh}\left(\frac{1}{2}\right)\right) .
$$

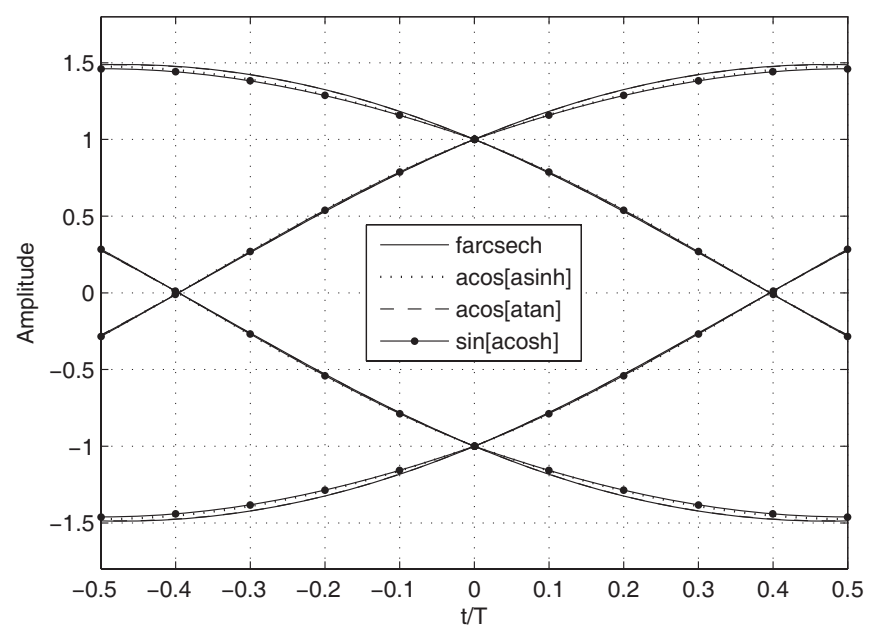

Fig. 3. Inner and outer contour boundaries of eye diagrams with roll-off factor $\alpha=0.35$.

TABLE I

Eye Opening And Maximum Distortion Of Eye Diagrams

\begin{tabular}{c||cc} 
pulse & eye opening & max distortion \\
\hline \hline farcsech & 0.5983 & 1.4880 \\
acos[asinh] & 0.5680 & 1.4754 \\
acos[atan] & 0.5781 & 1.4863 \\
$\sin [\operatorname{acosh}]$ & 0.5999 & 1.4608 \\
\hline \hline
\end{tabular}

The frequency and time responses of all the considered pulses are illustrated in Fig. 1 and 2, respectively. Unfortunately, there are no closed-form expressions for the latter and hence their evaluation was carried out numerically via an inverse Fourier transform. The common characteristic of $S_{1}(f)-S_{4}(f)$ is their concave shape in the interval $B(1-\alpha)<$ $|f| \leq B$ and convex in $B<|f| \leq B(1+\alpha)$, so that an amount of energy is transferred in the high spectral region. Under these circumstances and after observing that the frequency responses are continuous functions but their first derivatives are discontinuous, it is found that the impulse responses' tails asymptotically decay as $1 / t^{2}[5$, Theorem 1$]$. We recall that both $\mathrm{RC}$ and fsech pulses have a decay rate as $1 / t^{3}$. However, a lower decay rate is not always a disadvantage as long as the amplitudes of the largest two sidelobes are smaller [2], [4]. From inspection of Fig. 2, it can be inferred that the strongest first-sidelobe suppression is offered by acos[asinh] whereas farcsech yields the worst performance in this aspect.

\section{Performance Evaluation}

In this section, we evaluate the performance of the proposed pulses from different practical perspectives of interest. In this light, we begin with the comparison of the eye diagrams which are a means of visually assessing the vulnerability of transmission systems to the problem of ISI [7]. For the sake of clarity, only the inner and outer contour boundaries, corresponding to minimum and maximum distortion, have been generated (see Fig. 3). This graph shows that $\sin [\mathrm{acosh}]$ performs remarkably better than any other pulse under investigation while acos[atan] and farcsech are almost identical. A quantitative insight can also be obtained after estimating the 
TABLE II

Bit Error Probabilities For $N=2^{9}$ InTERFERING Symbols AND SNR $=15$ DB

\begin{tabular}{c||c||cccc}
\hline$\alpha$ & pulse & $t / T= \pm 0.05$ & $t / T= \pm 0.1$ & $t / T= \pm 0.2$ & $t / T= \pm 0.3$ \\
\hline \hline \multirow{4}{*}{0.25} & farcsech & $5.3996 \mathrm{e}-8$ & $1.1011 \mathrm{e}-6$ & $2.8405 \mathrm{e}-4$ & $1.2496 \mathrm{e}-2$ \\
& acos[asinh] & $5.1488 \mathrm{e}-8$ & $9.9816 \mathrm{e}-7$ & $2.4946 \mathrm{e}-4$ & $1.1349 \mathrm{e}-2$ \\
& acos[atan] & $5.3114 \mathrm{e}-8$ & $1.0636 \mathrm{e}-6$ & $2.7117 \mathrm{e}-4$ & $1.2083 \mathrm{e}-2$ \\
& sin[acosh] & $5.2941 \mathrm{e}-8$ & $1.0565 \mathrm{e}-6$ & $2.6878 \mathrm{e}-4$ & $1.2003 \mathrm{e}-2$ \\
\hline \multirow{3}{*}{0.35} & farcsech & $3.5970 \mathrm{e}-8$ & $4.4580 \mathrm{e}-7$ & $7.6203 \mathrm{e}-5$ & $4.6950 \mathrm{e}-3$ \\
& acos[asinh] & $3.4124 \mathrm{e}-8$ & $4.0410 \mathrm{e}-7$ & $6.7653 \mathrm{e}-5$ & $4.2252 \mathrm{e}-3$ \\
& acos[atan] & $3.5310 \mathrm{e}-8$ & $4.3008 \mathrm{e}-7$ & $7.2778 \mathrm{e}-5$ & $4.5109 \mathrm{e}-3$ \\
& $\sin [\operatorname{acosh}]$ & $3.5182 \mathrm{e}-8$ & $4.2722 \mathrm{e}-7$ & $7.2196 \mathrm{e}-5$ & $4.4787 \mathrm{e}-3$ \\
\hline \multirow{3}{*}{0.5} & farcsech & $2.1875 \mathrm{e}-8$ & $1.4916 \mathrm{e}-7$ & $1.5344 \mathrm{e}-5$ & $1.2253 \mathrm{e}-3$ \\
& acos[asinh] & $2.0758 \mathrm{e}-8$ & $1.3617 \mathrm{e}-7$ & $1.4609 \mathrm{e}-5$ & $1.2202 \mathrm{e}-3$ \\
& acos[atan] & $2.1462 \mathrm{e}-8$ & $1.4410 \mathrm{e}-7$ & $1.4953 \mathrm{e}-5$ & $1.2093 \mathrm{e}-3$ \\
& $\sin [\operatorname{acosh}]$ & $2.1386 \mathrm{e}-8$ & $1.4323 \mathrm{e}-7$ & $1.4911 \mathrm{e}-5$ & $1.2092 \mathrm{e}-3$ \\
\hline \hline
\end{tabular}

eye opening of all pulses at a timing offset of $t / T=0.5$, as described in [8], with the corresponding results being given in Table I.

Consistent conclusions can be drawn after computing the maximum distortion experienced by each separate pulse which is, in general, a more quantitative measure of performance (see column 3, Table I). From a mathematical viewpoint, the maximum distortion is the magnitude of the largest possible ISI sample at any given time instant. In all cases, the point of maximum distortion occurs at $t / T= \pm 0.5$ and the minimum maximum distortion is again offered by the $\sin$ [acosh] pulse whereas farcsech is marginally outperformed by both acos[asinh] and acos[atan].

The last step of the evaluation process comprises the computation of the average bit error rates (BERs) in the presence of time sampling errors. It should be remembered that the BER is the ultimate metric of performance assessment that includes the effects of noise, synchronization and distortion [8]. The error rates have been estimated according to [9] for binary antipodal signaling. A signal-to-noise ratio (SNR) of $15 \mathrm{~dB}$ has been assumed while $2^{9}$ interfering symbols are generated for all pulses. The obtained results are tabulated in Table II.

Generally speaking, timing jitter raises BER values since ISI is a result of the receiver eye being sampled off center [2]. It can be easily noticed that all the proposed pulses perform always better than the farcsech pulse. More importantly, acos[asinh] offers the lowest BER for 11 of the 12 cases under consideration. The only exception occurs for $\alpha=0.5$ and $t / T= \pm 0.3$ where acos[asinh] returns a slightly higher BER compared to acos[atan] and $\sin [$ acosh]. This can be attributed to the slower decay of the weaker higher-order sidelobes of the former which results in an increased ISI and consequently worse BER for large values of roll-off factor and symbol timing errors. Summarizing, it is apparent that, between the proposed pulses, acos[asinh] yields the best performance in terms of BER for the great majority of cases, while sin[acosh] is superior in terms of eye opening and maximum distortion.

\section{CONCLUSION}

In this letter, three alternative Nyquist pulses were devised that exploit the inherent flexibility of using the concepts of inner and outer functions. The proposed pulses were compared in detail with the farcsech pulse which is the most sophisticated two-parameter pulse available in the literature. It was clearly demonstrated that in terms of BER and maximum distortion an improved performance is systematically achieved, with acos[asinh] and $\sin [$ acosh] returning respectively the best results in these two key categories.

\section{REFERENCES}

[1] H. Nyquist, "Certain topics in telegraph transmission theory" AIEE Trans., vol. 47, pp. 617-644, 1928.

[2] N. C. Beaulieu, C. C. Tan, and M. O. Damen, "A "better than" Nyquist pulse," IEEE Commun. Lett., vol. 5, no. 9, pp. 367-368, Sept. 2001.

[3] X. Xia, "A family of pulse-shaping filters with ISI-free matched and unmatched filter properties," IEEE Trans. Commun., vol. 45, no. 10, pp. 1157-1158, Oct. 1997.

[4] A. Assalini and A. M. Tonello, "Improved Nyquist pulses," IEEE Commun. Lett., vol. 8, no. 2, pp. 87-89, Feb. 2004.

[5] N. C. Beaulieu and M. O. Damen, "Parametric construction of Nyquist-I pulses," IEEE Trans. Commun., vol. 52, no. 12, pp. 2134-2142, Dec. 2004.

[6] S. D. Assimonis, M. Matthaiou, and G. K. Karagiannidis, "Improved parametric families of ISI-free Nyquist pulses using inner and outer functions," submitted to IEEE Trans. Commun., July 2008.

[7] J. G. Proakis, Digital Communications, 4th ed. New York: McGraw-Hill, 2000.

[8] C. C. Tan and N. C. Beaulieu, "Transmission properties of conjugate-root pulses," IEEE Trans. Commun., vol. 52, no. 4, pp. 553-558, Apr. 2004.

[9] N. C. Beaulieu, "The evaluation of error probabilities for intersymbol and cochannel interference," IEEE Trans. Commun., vol. 31, pp. 1740-1749, Dec. 1991. 